\title{
Different Clinical Courses with Severe Postpartum Coagulopathy in Two Cases Showing the Same Histological Findings those Local Amniotic Fluid Emboli
}

\begin{abstract}
Keywords: Amniotic fluid embolism; Mucin thrombus; Uterine atony; Retained placenta

\section{Abstract}

We report two cases of postpartum intractable bleeding with coagulopathy.

Case 1: A 34-year-old low-risk woman at 39 weeks of gestation was admitted to our hospital. After an uneventful vaginal delivery, placenta separation failed. Ultrasonography revealed absence of placental lacunae suggesting a retained placenta with a lower possibility of placenta accreta. Manual separation was then attempted in the operating room with success. However, profound vaginal bleeding continued despite hemostasis with intrauterine balloon tamponade and oxytocin administration. She eventually underwent a subtotal hysterectomy. Histological examination showed amniotic fluid emboli in the uterine corpus and no placental tissue.

Case 2: A 30-year-old woman at 38 weeks of gestation was referred due to atonic bleeding after a vacuum delivery. Enhanced computed tomography showed diffuse intravenous contrast extravasation within the uterus. Profound vaginal bleeding continued despite hemostasis with uterine artery embolization. She eventually underwent a subtotal hysterectomy. Histological examination showed amniotic fluid emboli in the uterine corpus. The presented cases had evident etiologies such as retained tissue or uterine atony those rarely need to invasive procedures for hemostasis, but eventually led to intractable bleeding, in which mucin emboli were confirmed in the uterine corpus of both cases. We should not exclude the possibility of potential mild amniotic fluid embolism among the group of postpartum intractable bleeding caused by any etiologies.
\end{abstract}

\section{Introduction}

According to an autopsy registry survey in Japan, half of maternal deaths are caused by obstetric hemorrhage excepting amniotic fluid embolism (AFE) [1]. These mainly involve DIC related to pregnancyinduced hypertension, birth canal tears, atonic bleeding, and abnormal placental adherence.

Generally, the cases of postpartum hemorrhage with above etiologies can be sufficiently treated with blood transfusion, administration of uterotonic agents, bimanual compression of uterus, and uterine packing. Few cases are needed to invasive procedures such as hysterectomy for hemostasis [2]. Therefore, some additional factors might be involved in developing to refractory cases.

AFE develops after the entry of fetal antigen into maternal circulation. The entry of amniotic fluid may cause an immunological reaction including complement activation, anaphylaxis, and inflammatory response followed by developing to respiratory failure,

\section{Journal of}

Syndromes

\author{
Yukiko Matsuzawa1, Seishi Furukawa ${ }^{1 *}$, Chikage \\ Kiyomoto', Masachika Fujiwara ${ }^{2}$, Makoto \\ Mochizuki ${ }^{2}$ and Mitsutoshi Iwashita ${ }^{1}$ \\ ${ }^{I}$ Department of Obstetrics \& Gynecology, School of Medicine, \\ Kyorin University, Tokyo, Japan \\ ${ }^{2}$ Department of Pathology, School of Medicine, Kyorin University, \\ Tokyo, Japan \\ *Address for Correspondence \\ Seishi Furukawa, MD, Department of Obstetrics \& Gynecology, \\ School of Medicine, Kyorin University, 6-20-2 Shinkawa, Mitaka, \\ Tokyo, 181-8611, Japan, Tel: 81-0422-47-5511; Fax: 81-0422-47- \\ 3177; E-mail: shiiba46seishi@gmail.com \\ Submission: 24 April, 2016 \\ Accepted: 10 June, 2016 \\ Published: 15 June, 2016 \\ Copyright: $\odot 2016$ Matsuzawa Y, et al. This is an open access article \\ distributed under the Creative Commons Attribution License, which \\ permits unrestricted use, distribution, and reproduction in any medium, \\ provided the original work is properly cited.
}

cardiac arrest, and disseminated intravascular coagulation $[3,4]$. The local entry of amniotic fluid may also cause an immunological reaction in the uterus followed by developing to a tonic bleeding and resulting in refractory to conventional therapies [5]. Then, refractory cases are considered as mild AFE [5]. In fact, mild AFE is not rare event [6].

The causes of obstetric hemorrhage such as operative deliveries, eclampsia, polyhydramnios, cervical lacerations, or uterine rupture are also reported as possible causes for the development of AFE [7]. Therefore, mild AFE may play an important role for developing to refractory case among obstetrical hemorrhage caused by other etiologies.

In this report, we present two cases of intractable bleeding with coagulopathy involving different clinical courses, in which amniotic fluid emboli were confirmed in the uterine corpus of both cases. One case was uterine atony and the other involved retained placental tissue without abnormal placental attachment to the myometrium. In general, consumptive coagulopathy or disseminative intravascular coagulation due to evident etiologies should be excluded from entry criteria for clinical diagnosis of AFE [5,8]. Accordingly, our cases might be failed to include the category for AFE. We discuss the involvement of potential mild AFE for intractable bleeding with evident etiology.

\section{Case Report}

We obtained informed consent for case report when the patient was in hospital.

\section{Case 1}

A 34-year-old, gravida 1, para 1, low-risk woman with one vaginal delivery was admitted to our hospital at 38 weeks of gestation in labor. Her past medical history was unremarkable. Her labor process was going well and resulted in the uneventful vaginal delivery of a male infant weighing $3423 \mathrm{~g}$. However, the placenta failed to 
Citation: Matsuzawa Y, Furukawa S, Kiyomoto C, Fujiwara M, Mochizuki M, et al. Different Clinical Courses with Severe Postpartum Coagulopathy in Two Cases Showing the Same Histological Findings those Local Amniotic Fluid Emboli. J Syndromes. 2016;3(1): 4.

separate despite uterine massage. Ultrasonography on site revealed a posterior placenta and no placental lacunas were observed. We made a tentative diagnosis of a retained placenta, and manual separation was then attempted in the operating room under general anesthesia. The manual separation succeeded, but a heavy vaginal bleeding started immediately after the operation. We attempted hemostasis with intrauterine balloon tamponade and $250 \mathrm{ml}$ of saline infusion for 30 minutes. The patient was also resuscitated with freshly frozen plasma, packed red cells, and vasoactive agents to promote uterine contraction. However, her clotting parameters were worsened with increased Activated Partial Thromboplastin Time (APTT; $51.1 \mathrm{sec}$.) and decreased fibrinogen $(111 \mathrm{mg} / \mathrm{dl})$. The heavy vaginal bleeding continued despite hemostasis, and the patient finally underwent a subtotal hysterectomy. During the peripartum course, 30 units of packed red blood cells, 30 units of freshly frozen plasma, and 20 units of platelet concentrate were infused. Estimated blood loss was 8000 $\mathrm{ml}$. Postoperatively, she was hemodynamically stable and resulting in extubation on the first day after surgery. Histological examination of the resected uterus revealed a few microthromboses in small vessels of the uterine corpus and no placental tissue remaining. In addition, a few amniotic fluid emboli were confirmed in small vessels of the uterine corpus (Figure 1).

\section{Case 2}

A 30-year-old, gravida 2, para 2, healthy woman was admitted to a private clinic for labor induction at 38 weeks of gestation. She showed lower extremity edema without proteinuria before admission. Her past medical history was unremarkable. Six hours after uterine stimulation with oxytocin augmentation, she showed a cervix dilatation of $8 \mathrm{~cm}$ and rupture of the membrane. She simultaneously lost consciousness for 3 minutes with preserved blood pressure $(111 / 64 \mathrm{mmHg})$. Fetal bradycardia $(60 \sim 80 \mathrm{bpm})$ also occurred and lasted for at least 13 minutes. A female infant weighing $3380 \mathrm{~g}$ was subsequently delivered by vacuum extraction. Immediately after separation of the placenta, a heavy vaginal bleeding started. The woman presented hypotension with $65 \mathrm{mmHg}$ of systolic blood pressure. She was resuscitated by aggressive administration of crystalloid and vasoactive agents. She underwent uterine packing for hemostasis. The patient was then immediately transferred to the emergency department of our hospital with atonic bleeding after the vacuum delivery. On arrival, the blood pressure and heart rate were 126/33 $\mathrm{mmHg}$ and $134 \mathrm{bpm}$, respectively. Her clotting parameters had been altered with increased APTT ( $\geq 240 \mathrm{sec}$.) and decreased fibrinogen $(<10 \mathrm{mg} / \mathrm{dl})$. Vaginal examination showed uterine atony with small vaginal laceration. Vaginal bleeding continued. In order to identify the source of bleeding, enhanced computed tomography was performed and revealed a diffuse intravenous contrast extravasation within the uterus. Despite hemostasis with uterine artery embolization, the profound vaginal bleeding continued. She eventually underwent a subtotal hysterectomy. During the peripartum course, estimated blood loss was $8300 \mathrm{ml} 46$ units of packed red blood cells, 44 units of freshly frozen plasma, and 40 units of platelet concentrate were infused. Postoperatively, she was hemodynamically stable and resulting in extubation on the second day after surgery. Histological examination of the resected uterus revealed a few amniotic fluid emboli in the uterine corpus just beneath the endometrium (Figure 2). Small specimen of placental tissue was retained on the endometrium with decidual change. Interestingly, concentrations of zinc coproporphyrin I ( $\mathrm{ZnCP}-\mathrm{I})$ and serum sialyl Tn antigen $(\mathrm{STN})$ were not elevated $(<1.6 \mathrm{pmol} / \mathrm{ml}$ and $32.0 \mathrm{U} / \mathrm{ml}$, respectively). ZnCP-I and STN are characteristic components in meconium and are introduced as a useful serum test for diagnosis of AFE $[9,10]$.

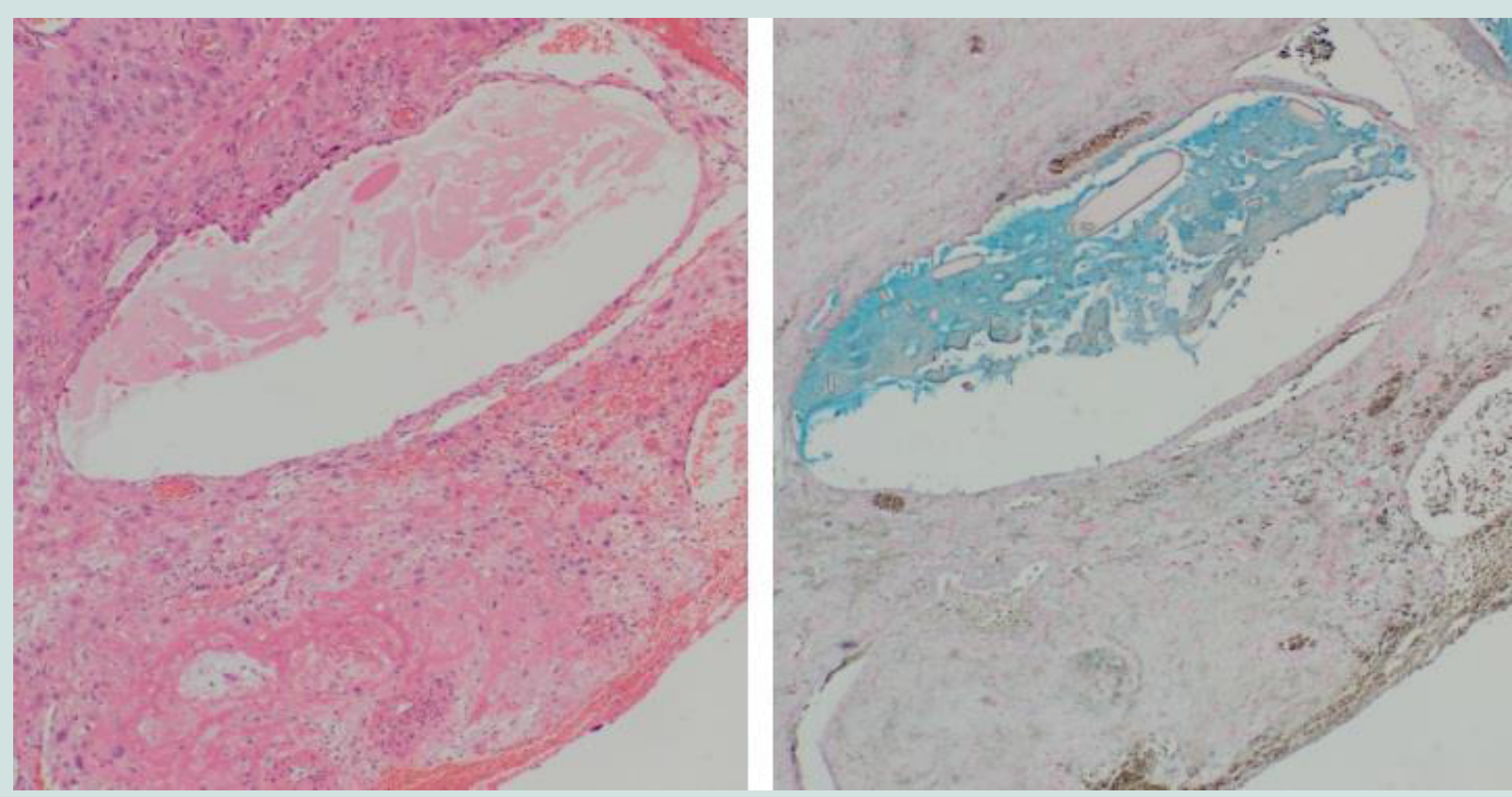

Figure 1: Histological section of the resected uterus showing Alcian-blue-stain-positive amniotic fluid embolus in the small vessel (left: H\&E×200, right: Alcian bluex200). 

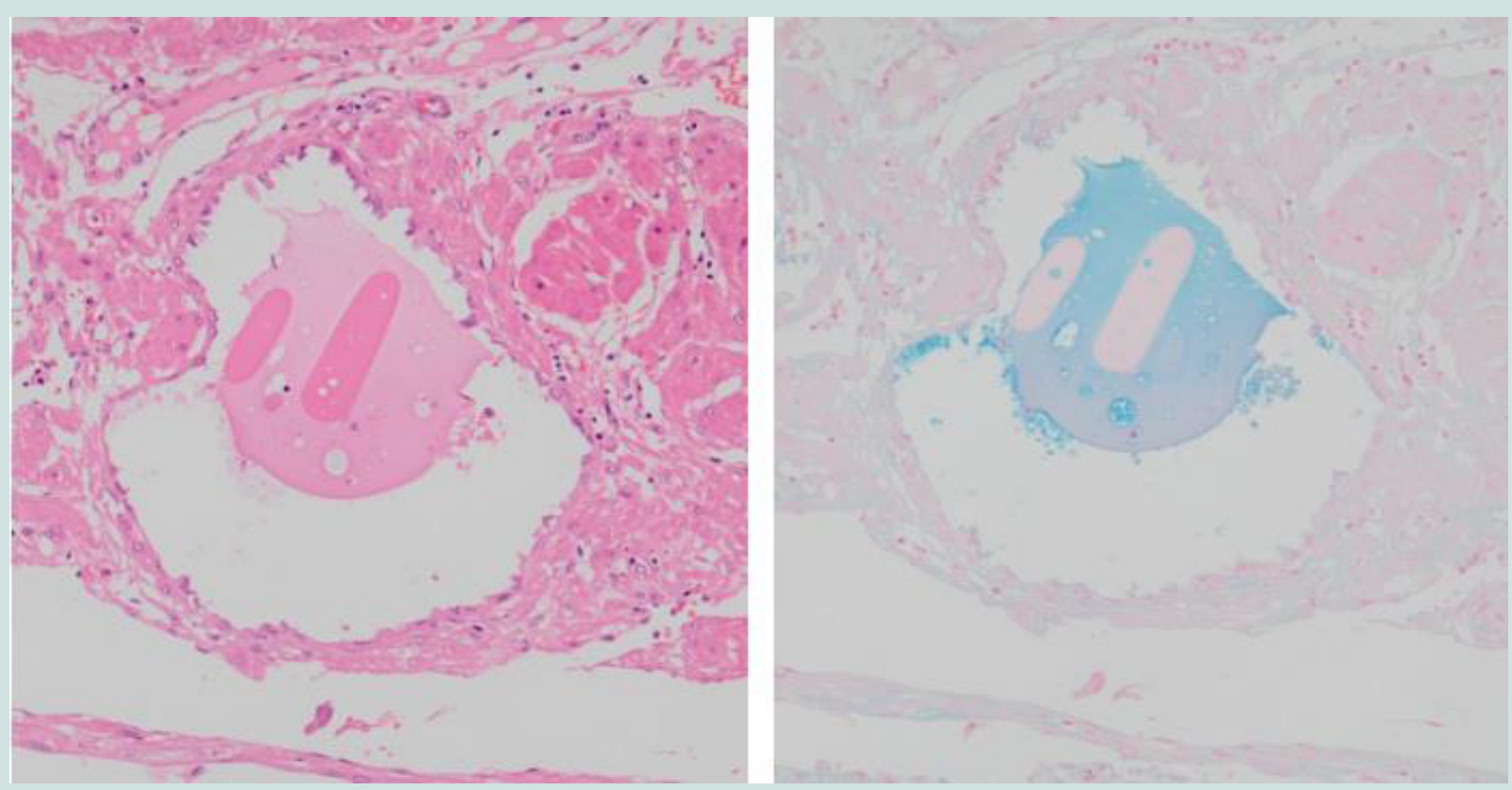

Figure 2: Histological section of the resected uterus showing Alcian-blue-stain-positive amniotic fluid embolus in the small vessel beneath the endometrium (left: H\&E×200, right: Alcian blue×200).

\section{Discussion}

In the current report, we presented two cases that finally led to intractable bleeding with disseminative intravascular coagulation through different clinical courses, in which amniotic fluid emboli were confirmed in the uterine corpus of both cases. Clinical diagnosis in one case involved retained placental tissue, and uterine atony in the other case. Generally, consumptive coagulopathy or disseminative intravascular coagulation due to evident etiologies should be excluded from entry criteria for clinical diagnosis of AFE [5,8]. Therefore, our cases might be regarded as exclusion cases for AFE. However, the cases of postpartum hemorrhage with both etiologies can be sufficiently treated with less invasive therapy [2]. In other words, few cases are needed to invasive procedures. Therefore, another factor might be involved in developing to intractable bleeding with our cases. Subsequently, we assumed the involvement of mild AFE in addition to primary etiology for obstetrical hemorrhage.

The presented cases had no other clinical manifestations including lung, liver, heart, or renal failure. In addition, there were no elevations of ZnCP-I and STN in case 2. Therefore, our presented cases might be representing mild AFE. In the autopsy series of AFE, a component of amniotic fluid was observed in various organs such as the lung, heart, kidney, spleen, pancreas, brain and uterus [11]. It is reported that the number of failed organs associated with AFE is significantly lower for survivors compared with non-survivors [12]. Therefore, clinical manifestations may depend on the number of failed organs. Mild AFE may only show intractable bleeding as a clinical manifestation. Thus, we may miss the involvement of mild AFE under the presence of primary etiology for pregnancy-related coagulopathy.

The factor concerning prerequisites for the development of mild AFE may be some kind of lacerations. Yang et al. reported that amniotic fluid debris was found in the laceration site vasculature of the endocervix in the removed uterus [13]. The case was initially treated as isolated coagulopathy after an uneventful delivery. Mathelier et al. reported that AFE may develop suddenly after a previous cesarean section with placenta previa and accrete [14]. They postulated that the presence of abnormal placentation easily provides the laceration for amniotic fluid to enter the systemic circulation, causing profound bleeding or cardiopulmonary arrest. However, there was no evidence of placenta accrete or obvious laceration in the removed uterus in case 1 of our study. No previous reports have shown the possibility of a retained placenta developing amniotic fluid embolism-related coagulopathy until now. The passage of fetal material may occur easily in cases involving a large amount of retained tissue and poor uterine contraction.

To date, the presence of fetal or amniotic components in the maternal circulation is no longer a sufficient condition developing to amniotic fluid embolism [3,4]. However, the presence of fetal or amniotic components in the maternal circulation is a necessary condition for developing to amniotic fluid embolism. In order to get closer to precise diagnosis for suspected cases, the inspection of obtained tissue for detection of amniotic fluid components is primal manner and definitely needed. If the absence of fetal components is confirmed by inspection, the case is less likely to be AFE. Nevertheless it is unclear the difference between women those develop the fatal reaction and women those do not. It is currently thought that the AFE develops after a complex reaction based on the type of fetal components, the amount of fetal components, and immunological reaction of maternal including complement activation, anaphylaxis, and inflammatory response $[3,4,15]$. We also experienced a case of AFE with massive platelet aggregations in pulmonary capillaries [16], suggesting platelet-activating factor (PAF) might have involved in. Therefore, laboratory test including the measurement of serum tryptase, complement, or PAF activity might be helpful to support 
Citation: Matsuzawa Y, Furukawa S, Kiyomoto C, Fujiwara M, Mochizuki M, et al. Different Clinical Courses with Severe Postpartum Coagulopathy in Two Cases Showing the Same Histological Findings those Local Amniotic Fluid Emboli. J Syndromes. 2016;3(1): 4.

the diagnosis of AFE. Unfortunately, presented two cases only did the examination for amniotic fluid components.

The precise mechanism of intractable bleeding with disseminative intravascular coagulation in mild AFE like our cases is not completely understood. Recently, Farhana et al. reported that local immune reactions cause postpartum acute myometritis followed by uterine atony with postpartum hemorrhage [17]. One of the candidates for postpartum acute myometritis is amniotic fluid emboli. Coagulopathy may then occur if amniotic fluid emboli are present in the uterine corpus without obvious lacerations in the uterus. Although a comprehensive understanding of the pathophysiological mechanism is lacking, histological examination including immunohistochemical studies is definitely needed to reveal the precise mechanism in cases of intractable bleeding requiring hysterectomy.

AFE is widely recognized as an unpredictable and severe complication of pregnancy. Its incidence is approximately 1 in 40,000 deliveries [18]. Currently, AFE remains a clinical diagnosis [19] and the incidence may increase if cases of potential mild AFE with primary etiology for pregnancy-related coagulopathy like our cases are included. We must pay attention to potential mild AFE in case of severe pregnancy-related coagulopathies even if the presence of evident etiologies.

\section{References}

1. Kanayama N, Inori J, Ishibashi-Ueda H, Takeuchi M, Nakayama M, et al (2011) Maternal death analysis from the Japanese autopsy registry for recent 16 years: significance of amniotic fluid embolism. J Obstet Gynaecol Res 37: $58-63$

2. Furuta K, Furukawa S, Hirotoshi U, Michikata K, Kai K, et al. (2014) Differences in maternal morbidity concerning risk factors for obstetric hemorrhage. Austin J Obstet Gynecol 1: 5.

3. Benson MD (2012) Current concepts of immunology and diagnosis in amniotic fluid embolism. Clin Dev Immunol 2012: 7

4. Turillazzi E, Neri M, Bello S, Riezzo I, Fineschi V (2014) Amniotic fluid embolism: moving diagnosis through the time. From the mechanical pulmonary vascular occlusion until an immuno - inflammatory pathogenesis? Curr Pharm Biotechnol 14: 1179-1188.
5. Kanayama N, Tamura N (2014) Amniotic fluid embolism: pathophysiology and new strategies for management. J Obstet Gynaecol Res 40: 1507-1517.

6. Shen F, Wang L, Yang W, Chen Y (2016) From appearance to essence: 10 years review of atypical amniotic fluid embolism. Arch Gynecol Obstet 293: 329-334.

7. Conde-Agudelo A, Romero R (2009) Amniotic fluid embolism: an evidencebased review. Am J Obstet Gynecol 201: 445.e1-e13.

8. Knight M, Berg C, Brocklehurst P, Kramer M, Lewis G, et al. (2012) Amniotic fluid embolism incidence, risk factors and outcomes: a review and recommendations. BMC Pregnancy Childbirth 12: 7.

9. Kanayama N, Yamazaki T, Naruse H, Sumimoto K, Horiuchi K, et al. (1992) Determining zinc coproporphyrin in maternal plasma--a new method for diagnosing amniotic fluid embolism. Clin Chem 38: 526-529.

10. Oi H, Kobayashi H, Hirashima Y, Yamazaki T, Kobayashi T, et al. (1998) Serological and immunohistochemical diagnosis of amniotic fluid embolism. Semin Thromb Hemost 24: 479-484.

11. Liban E, Raz S (1969) A clinicopathologic study of fourteen cases of amniotic fluid embolism. Am J Clin Pathol 51: 477-486.

12. Matsuda Y, Kamitomo M (2009) Amniotic fluid embolism: a comparison between patients who survived and those who died. J Int Med Res 37: 15151521.

13. Yang JI, Kim HS, Chang KH, Ryu HS, Joo HJ (2006) Amniotic fluid embolism with isolated coagulopathy: a case report. J Reprod Med 51: 64-66.

14. Mathelier AC, Karachorlu K (2006) Placenta previa and accreta complicated by amniotic fluid embolism. Int J Fertil Womens Med 51: 28-32.

15. West $M$ (2016) Amniotic fluid embolism: a historical perspective in diagnosis and management. BJOG 123: 110.

16. Furukawa S, Urabe H, Nagai Y, Sameshima H, Ikenoue T, et al. (2010) A rare case of amniotic fluid embolism with massive platelet aggregations in pulmonary capillaries. J Obstet Gynaecol Res 36: 397-400.

17. Farhana M, Tamura N, Mukai M, Ikuma K, Koumura Y, et al. (2015) Histological characteristics of the myometrium in the postpartum hemorrhage of unknown etiology: a possible involvement of local immune reactions. J Reprod Immunol 110: 74-80.

18. Clark SL (2014) Amniotic fluid embolism. Obstet Gynecol 123(2 Pt 1): 337348.

19. Society for Maternal-Fetal Medicine (SMFM) with the assistance of. Electronic address: pubs@smfm.org, Pacheco LD, Saade G, Hankins GD, Clark SL (2016) Amniotic fluid embolism: diagnosis and management. Am J Obstet Gynecol [Epub ahead of print]

\section{Acknowledgements}

We thank Dr. N. Kanayama of the Department of Obstetrics and Gynecology, Hamamatsu University School of Medicine, for examining the serum level of zinc coproporphyrin I (ZnCP-I) and sialyl Tn antigen. 